\title{
Survey of serious adverse events and safety evaluation of oral anticancer drug treatment in Japan: A retrospective study
}

\author{
KENJI KAWASUMI $^{1,2}$, AZUSA KUJIRAI ${ }^{2}$, REIKO MATSUI ${ }^{1}$, YOHEI KAWANO ${ }^{2}$, \\ MASAKAZU YAMAGUCHI ${ }^{3}$ and TAKAO AOYAMA ${ }^{2}$ \\ ${ }^{1}$ Department of Pharmacy, National Cancer Center Hospital East, Kashiwa, Chiba 277-8577; \\ ${ }^{2}$ Faculty of Pharmaceutical Sciences, Tokyo University of Science, Noda, Chiba 278-8511; \\ ${ }^{3}$ Department of Pharmacy, National Cancer Center Hospital, Tokyo 104-0045, Japan
}

Received March 15, 2020; Accepted September 22, 2020

DOI: $10.3892 / \operatorname{mco} .2020 .2174$

\begin{abstract}
The present study assessed the safety of outpatient oral anticancer chemotherapeutic drugs by investigating the type and frequency of serious adverse effects (SAEs). Emergency hospitalization, unplanned consultations and telephone calls were investigated in 1,832 patients who received oral anticancer drug treatment at the National Cancer Center Hospital East between December 1, 2014 and November 30,2015. Oral cytotoxic anticancer and molecular targeted drugs were administrated to $1,140(62.2 \%)$ and 692 (37.8\%) patients, respectively. A total of 52 (2.8\%) SAEs were reported, with $32(2.8 \%)$ occurring following cytotoxic anticancer drug administration and $20(2.9 \%)$ occurring after molecular targeted drug treatment. The most common SAE was gastrointestinal toxicity. The median time to SAE occurrence was 32 days (range, 5-1,705 days). The rate of unplanned consultations and telephone calls were 5.5 and $37.9 \%$ among all patients, respectively, with skin reactions being the most common reason for unplanned consultations. SAEs often occurred early after treatment initiation. It was concluded that measures against gastrointestinal toxicity are particularly important were administering chemotherapeutic agents.
\end{abstract}

\section{Introduction}

Recently, outpatient cancer chemotherapy has become an increasingly popular option. The number of patients opting for outpatient oral anticancer drug monotherapy and combination therapy with oral and injectable drugs has been increasing (1-3). Oral anticancer drug treatment can continue while patients live at home and without possible injection site

Correspondence to: Mr. Kenji Kawasumi, Department of Pharmacy, National Cancer Center Hospital East, 6-5-1 Kashiwanoha, Kashiwa, Chiba 277-8577, Japan

E-mail: kkawasum@east.ncc.go.jp

Key words: oral anticancer drugs, serious adverse event, emergency hospitalization, unplanned consultation, telephone calls complications or the constraints of drip time that accompanies injectable anticancer drugs, thus increasing the patients' quality of life (4). However, as with injectable anticancer drugs, patients on oral anticancer drugs must be carefully monitored for hematotoxicity and gastrointestinal toxicity. For patients on molecular targeted anticancer drugs, various side effects, such as skin reactions, hyperglycemia or interstitial pneumonitis, may occur. When injectable anticancer drug treatment is administered at a hospital, doctors can confirm the patient's general condition prior to administration, and nurses or pharmacists can do so during administration. Conversely, for oral anticancer drug treatment at home, medication management and monitoring for side effects must be performed by the patients or their family members; thus, it is necessary that they fully understand the potential side effects and safety concerns.

To evaluate the safety of outpatient chemotherapy, emergency (unplanned) hospitalization rates due to serious adverse events (SAEs) caused by chemotherapy were used (5). In cases of emergency hospitalization due to an SAE, discontinuation or postponement of planned treatment, decreases in the chemotherapeutic dose and increases in medical expenses due to hospitalization have all been reported (6). In a survey of outpatient cancer chemotherapy safety for major cancer treatment hospitals in Japan, the SAE frequency was 0.4-2.8\%, suggesting that outpatient chemotherapy can be safely performed (7). Although treatments with oral anticancer drugs was limited in Japan in 2009, it has been reported that the SAE frequency for the oral chemotherapeutic regimens, including tegafur/oteracil/gimeracil (S-1), was 3.3-5.0\%, higher than that of injectable anticancer drugs, suggesting the need for an urgent response (7).

The frequency of SAEs in patients receiving chemotherapy has been reported to be higher in clinical practice compared with clinical trials, as reported in a systematic review of lung cancer treatment (8). Therefore, it is important to clarify the frequency and details of SAEs in actual clinical practice. Studies from other countries have reported that the safety of oral anticancer drug treatment is improved when pharmacists or nurses monitor the patients (9-12). There have been reports of pharmacist-led patient education programs to reduce adverse events $(9,10)$, improving the adherence of oral anticancer drugs (11), and reporting the usefulness of nursing 
interventions (12). As there are relatively fewer reports on oral anti-cancer interventions in Japan, limited information is available regarding these interventions for medical staff. In Japan, safety surveys have been primarily conducted for injectable anticancer drug therapies and there are relatively few similar reports for oral anticancer drugs $(13,14)$. Since 2009 , cancer treatment with oral anticancer drugs has become more common. With the increase in the use of orally administrable cytotoxic cancer drugs and molecular targeted drugs, safety evaluation of the current oral anticancer drug treatment in actual clinical practice may provide important information for future cancer treatments. Therefore, a safety assessment of the outpatients administered oral anticancer chemotherapy drugs was performed by investigating the nature and frequency of SAEs in patients receiving oral anticancer drug treatment in Japan. The differences in SAEs between cytotoxic cancer drugs and molecular targeted drugs was also compared in the present study. To the best of our knowledge, this is the first report on SAE occurrence in clinical practice during outpatient chemotherapy in Japan.

\section{Patients and methods}

Study population. Study subjects were patients who received oral anticancer drug therapy (single-agent or combination therapy) between December 1, 2014 and November 30, 2015 at the National Cancer Center Hospital East (Japan). Patients $<18$ years of age, patients involved in clinical trials, cases that could not be followed up, or patients receiving oral hormone monotherapy were excluded. The median age of patients was 64 (range 19-89) years with 1,031 (56.3\%) males and $801(43.7 \%)$ females.

Survey items. Information on age, sex, cancer type, performance status at the start of treatment, and setting and nature of previous therapy were collected as patient characteristics. The mechanism of action of chemotherapy was defined as a cytotoxic anticancer drug, which is a drug that acts on the cell cycle to directly cause DNA damage, or molecular target drug, a drug that acts on a target protein involved in cancer growth. Additional data collected included the reasons for treatment and the type of prescribed treatment, whether there was a causal relationship between anticancer drugs and emergency hospitalization or unplanned consultation, and the number and content of telephone calls from patients to the hospital. The definition of SAE was based on a previous report in Japan (7); an adverse event requiring hospitalization within the period of oral anticancer drug treatment that was causally related to the administered anticancer drug. The causality between a SAE and treatment with an anticancer drug was assessed by a physician and classified into 5 categories (unrelated, unlikely, possible, probable and definite) based on the guidelines of the National Cancer Institute (February 29, 2012) (15). Patients classified as possible, probable and definite were considered to have a causal relationship. Since the patients enrolled in this study were patients undergoing outpatient cancer chemotherapy, hematological toxicity was evaluated appropriately within the clinical practice. Therefore, even in emergency hospitalization as a result of a SAE, lymphopenia and non-febrile neutropenia were also evaluated and diagnosed. In addition, we also investigated infections that were determined to be causally related to anticancer drug treatment, based on the physician's diagnosis. If a causal relationship could not be judged from the patient's medical record, the reason for seeking unplanned medical attention was defined as an SAE if a strong correlation to anticancer drug treatment could be identified. The frequency of SAEs and the prescription numbers was calculated for each patient. Prescription numbers were defined as the ordered number of oral anticancer drugs at the time of the patient's visit. The time to SAE occurrence was defined as the time period between the start of oral anticancer drug treatment and the date of emergency hospitalization for SAE. The definition of unplanned consultation was a non-regular visit where a doctor judged an SAE caused by anticancer drugs as not necessary for hospitalization. The telephone consultations received from patients regarding cancer chemotherapy during the survey period were also assessed.

Statistical analysis. Statistical analysis was performed using SPSS version 23 (IBM, Corp.). Each characteristic between the SAE and non-SAE groups was performed using the Chi-square test or Fisher's exact test for categorical variables and Mann-Whitney $\mathrm{U}$ test for continuous variables. $\mathrm{P}<0.05$ was considered to indicate a statistically significant difference.

\section{Results}

Patient characteristics. The study population included 1,832 subjects and a total of 10,909 oral anticancer drug prescriptions. Patient characteristics are shown in Table I. The five most prevalent types of cancer amongst the cohort were lung cancer with 449 cases $(24.4 \%)$, colon cancer with 311 cases (16.9\%), gastric cancer with 306 cases $(16.6 \%)$, blood cancer with 138 cases $(7.5 \%)$ and breast cancer with 134 cases $(7.3 \%)$. A total of $1,341(73.2 \%)$ patients underwent oral anticancer monotherapy drug treatment and $491(26.8 \%)$ patients received injections of combined oral monotherapy drugs. Upon classification by mechanism of action, 1,140 (62.2\%) patients received cytotoxic anticancer drugs and 692 (37.8\%) received molecular targeted anticancer drugs. The treatment setting was $1,360(73.2 \%)$ for metastatic, and relapse and 472 (26.8\%) for pre- and post-operative treatments. There was no significant difference in patient characteristics between the SAE and non-SAE groups.

SAEs for oral anticancer drugs. The total number of SAEs was 52 (2.8\% of patients experienced an SAE); 32 (2.8\%) of those of the patients were on cytotoxic anticancer drugs and $20(2.9 \%)$ on molecular targeted anticancer drugs; 37 (2.7\%) received oral anticancer drug monotherapy and $15(2.8 \%)$ received injection combination therapy (Table II). Among patients receiving oral cytotoxic anticancer drugs with a high occurrence of SAE, $19(2.8 \%)$ were on S-1, $8(3.9 \%)$ were on capecitabine and 3 (3.9\%) were on trifluridine/tipiracil and experienced an SAE. Among patients receiving molecular targeted anticancer drugs, $4(9.5 \%)$ patients on afatinib, $3(15.8 \%)$ patients on axitinib and $3(4.8 \%)$ patients on regorafenib experienced an SAE. The majority of cases of emergency hospitalizations other than SAEs were cancer progression and infections that were not causally related to anticancer drugs. 
Table I. Patient characteristics of individuals receiving oral anticancer drug treatment.

\begin{tabular}{|c|c|c|c|c|}
\hline Characteristics & $\begin{array}{l}\text { All cases } \\
\mathrm{n}(\%)\end{array}$ & $\begin{array}{l}\text { SAE group } \\
\mathrm{n}(\%)\end{array}$ & $\begin{array}{c}\text { Non SAE group } \\
\mathrm{n}(\%)\end{array}$ & P-value \\
\hline All Cases & 1,832 & 52 & 1,780 & $\mathrm{~N} / \mathrm{A}$ \\
\hline Prescription number & 10,909 & N/A & $\mathrm{N} / \mathrm{A}$ & $\mathrm{N} / \mathrm{A}$ \\
\hline Age, years (median) & $64(19-89)$ & $66(32-78)$ & $66(19-89)$ & $0.975^{\mathrm{a}}$ \\
\hline Sex & & & & $0.480^{\mathrm{b}}$ \\
\hline Male & $1,031(56.3)$ & $32(61.5)$ & $999(56.1)$ & \\
\hline Female & $801(43.7)$ & $20(38.5)$ & 781 (43.9) & \\
\hline Cancer type & & & & $0.177^{\mathrm{b}}$ \\
\hline Lung cancer & $449(24.4)$ & $7(13.5)$ & $442(42.8)$ & \\
\hline Colorectal cancer & $311(16.9)$ & $12(23.1)$ & $299(16.8)$ & \\
\hline Gastric cancer & $306(16.6)$ & $12(23.1)$ & $294(16.5)$ & \\
\hline Hematological malignancy & $138 \quad(7.5)$ & $3(5.8)$ & 135 (7.6) & \\
\hline Breast cancer & $134 \quad(7.3)$ & $5 \quad(9.6)$ & $129(7.2)$ & \\
\hline Pancreatic cancer & $121 \quad(6.6)$ & $4(7.7)$ & 117 (6.6) & \\
\hline GIST & $104 \quad(5.6)$ & $0 \quad(0.0)$ & $104(5.8)$ & \\
\hline Head and neck cancer & $59 \quad(3.2)$ & $2(3.8)$ & $57 \quad(3.2)$ & \\
\hline Bladder cancer & $58 \quad(3.2)$ & $0 \quad(0.0)$ & $58 \quad(3.3)$ & \\
\hline Other & $103 \quad(5.6)$ & $2(3.8)$ & $101 \quad(5.7)$ & \\
\hline Treatment & & & & $0.736^{\mathrm{b}}$ \\
\hline Monotherapy & $1,341(73.2)$ & $37(71.2)$ & $1,304(73.3)$ & \\
\hline Injection combination therapy & $491(26.8)$ & $15(28.8)$ & $476(26.7)$ & \\
\hline Treatment setting & & & & $0.606^{\mathrm{b}}$ \\
\hline Metastatic/relapse & $1,360(73.2)$ & $37(71.2)$ & $1,323(74.3)$ & \\
\hline Cure (post/pre-operative treatment) & $472(26.8)$ & $15(38.8)$ & $457(25.7)$ & \\
\hline Performance status & & & & $0.112^{\mathrm{c}}$ \\
\hline 0 & $985(53.8)$ & $28(53.8)$ & $957(53.8)$ & \\
\hline 1 & $471(25.7)$ & $18(34.6)$ & $453(25.4)$ & \\
\hline 2 & 87 (4.7) & $5 \quad(9.6)$ & 82 (4.6) & \\
\hline 3 & $5 \quad(0.3)$ & $0 \quad(0.0)$ & $5 \quad(0.3)$ & \\
\hline 4 & $3 \quad(0.2)$ & $0 \quad(0.0)$ & $3 \quad(0.2)$ & \\
\hline Unknown & $281(15.3)$ & 1 (1.9) & $280(15.7)$ & \\
\hline Previously treated & & & & $0.131^{\mathrm{c}}$ \\
\hline $1 \mathrm{st}$ & $1,058(57.8)$ & $23(44.2)$ & $1,035(58.1)$ & \\
\hline 2nd & $303(16.5)$ & $10(19.2)$ & $293(16.5)$ & \\
\hline$>3 \mathrm{rd}$ & 471 & 19 (36.6) & $451(25.3)$ & \\
\hline Unknown & $1 \quad(0.1)$ & $0 \quad(0.0)$ & $1 \quad(0.1)$ & \\
\hline
\end{tabular}

${ }^{\mathrm{a}}$ Mann-Whitney U test. ${ }^{\mathrm{b}}$ Chi-square test. ${ }^{\mathrm{c}}$ Fisher's exact test. SAEs, serious adverse events; GIST, gastrointestinal stromal tumor.

SAEs in patients on cytotoxic anticancer drugs included $9(0.8 \%)$ reports of anorexia, $5(0.4 \%)$ reports of diarrhea and $4(0.3 \%)$ reports of dehydration. SAEs in patients on molecular targeted anticancer drugs included $7(1.0 \%)$ reports of diarrhea, $4(0.6 \%)$ reports of anorexia and $3(0.4 \%)$ reports of dehydration (Fig. 1). The nature of SAEs are shown in Table S1, and patients experienced $\leq 2$ types of SAEs consistently. For both types of anticancer drugs, SAEs due to gastrointestinal toxicity was the most common type of SAE. When the SAEs that occurred in this study were classified by Common Terminology Criteria for Adverse Events (CTCAE) v4.0, there was $1(1.9 \%)$ case of grade $1,9(17.9 \%)$ cases of grade 2 , $24(36.2 \%)$ cases of grade 3, $1(1.9 \%)$ case of grade 4 , and $17(32.7 \%)$ cases of unknown grading. A total of 25 patients $(48.1 \%)$ had serious symptoms of grade 3 or higher, which accounted for almost half of all cases.

The median time to SAE occurrence was 32 (range, 5-1,705) days for oral anticancer drugs. Based on the mechanism of action, cytotoxic anticancer drugs had a median time to SAE of 45 (range, 7-779) days and molecular targeted anticancer drugs a median time to SAE of 16 (range, 5-1,705) days (Fig. 2). When the time to SAE was binned into 0-30, 31-60, 
Table II. SAE incidence rates in patients receiving oral anticancer drugs according to mechanism of action per patient and prescription.

A, cytotoxic anticancer drugs

\begin{tabular}{|c|c|c|c|c|c|c|c|}
\hline Drug & Patients & Prescriptions & $\begin{array}{c}\text { Emergency } \\
\text { hospitalization }\end{array}$ & SAEs & $\begin{array}{c}\text { SAEs/ } \\
\text { patients }(\%)\end{array}$ & $95 \% \mathrm{CI}$ & $\begin{array}{c}\text { SAEs/ } \\
\text { prescriptions }(\%)\end{array}$ \\
\hline S-1 & 670 & 3,438 & 73 & 19 & 2.8 & $1.8-4.4$ & 0.6 \\
\hline Capecitabine & 203 & 1,180 & 19 & 8 & 3.9 & $1.9-7.7$ & 0.7 \\
\hline Tegafur/Uracil & 122 & 457 & 2 & 0 & 0.0 & $-0.6-3.7$ & 0.0 \\
\hline $\begin{array}{l}\text { Trifluridine/Tipyrazyl } \\
\text { hydrochloride }\end{array}$ & 77 & 346 & 19 & 3 & 3.9 & $0.9-11.3$ & 0.9 \\
\hline Etoposide & 14 & 70 & 5 & 1 & 7.1 & $-0.8-33.5$ & 1.4 \\
\hline Melphalan & 17 & 84 & 0 & 0 & 0.0 & $-3.2-21.6$ & 0.0 \\
\hline Hydroxycarbamide & 14 & 172 & 3 & 0 & 0.0 & $-3.6-25.2$ & 0.0 \\
\hline Cyclophosphamide & 10 & 44 & 0 & 0 & 0.0 & $-4.3-32.1$ & 0.0 \\
\hline Fludarabine & 4 & 5 & 1 & 1 & 25.0 & $3.4-71.1$ & 20.0 \\
\hline Mercaptopurine & 4 & 31 & 2 & 0 & 0.0 & $-5.6-54.6$ & 0.0 \\
\hline Procarbazine & 4 & 9 & 0 & 0 & 0.0 & $-5.6-54.6$ & 0.0 \\
\hline Temozomide & 1 & 5 & 1 & 0 & 0.0 & $-3.9-83.3$ & 0.0 \\
\hline Total & 1,140 & 5,841 & 125 & 32 & 2.8 & $2.0-4.0$ & 0.5 \\
\hline
\end{tabular}

B, Molecular targeted drugs

\begin{tabular}{|c|c|c|c|c|c|c|c|}
\hline Drug & Patients & Prescriptions & $\begin{array}{c}\text { Emergency } \\
\text { hospitalization }\end{array}$ & SAEs & $\begin{array}{c}\text { SAEs/ } \\
\text { patients }(\%)\end{array}$ & $95 \%$ CI & Prescriptions \\
\hline Gefitinib & 134 & 1,030 & 13 & 2 & 1.5 & $0.1-5.6$ & 0.2 \\
\hline Imatinib & 99 & 620 & 1 & 0 & 0.0 & $-0.8-4.5$ & 0.0 \\
\hline Erlotinib & 71 & 464 & 10 & 1 & 1.4 & $-0.5-8.3$ & 0.2 \\
\hline Regorafenib & 65 & 330 & 16 & 3 & 4.6 & $1.1-13.2$ & 0.9 \\
\hline Sorafenib & 48 & 353 & 3 & 0 & 0.0 & $-1.4-8.9$ & 0.0 \\
\hline Afatinib & 42 & 320 & 9 & 4 & 9.5 & $3.2-22.6$ & 1.3 \\
\hline Everolimus & 39 & 240 & 2 & 2 & 5.1 & $0.5-17.8$ & 0.8 \\
\hline Lenvatinib & 25 & 225 & 3 & 1 & 4.0 & $-0.9-21.1$ & 0.4 \\
\hline Sunitinib & 23 & 156 & 5 & 1 & 4.3 & $-0.9-22.7$ & 0.6 \\
\hline Lenalidomide & 23 & 152 & 3 & 1 & 4.3 & $-0.9-22.7$ & 0.7 \\
\hline Pazopanib & 19 & 108 & 1 & 1 & 5.3 & $-0.9-26.5$ & 0.9 \\
\hline Axitinib & 19 & 129 & 4 & 3 & 15.8 & $4.7-38.4$ & 2.3 \\
\hline Alectinib & 19 & 536 & 4 & 0 & 0.0 & $-3.0-19.8$ & 0.0 \\
\hline Crizotinib & 19 & 121 & 1 & 0 & 0.0 & $-3.0-19.8$ & 0.0 \\
\hline Nilotinib & 15 & 85 & 0 & 0 & 0.0 & $-3.5-23.9$ & 0.0 \\
\hline Thalidomide & 9 & 87 & 0 & 0 & 0.0 & $-4.6-34.5$ & 0.0 \\
\hline Lapatinib & 8 & 33 & 3 & 1 & 12.5 & $0.1-49.2$ & 3.0 \\
\hline Dasatinib & 7 & 46 & 1 & 0 & 0.0 & $-5.0-40.4$ & 0.0 \\
\hline Tretinoin & 4 & 13 & 0 & 0 & 0.0 & $-5.6-54.6$ & 0.0 \\
\hline Tamibarotene & 2 & 3 & 0 & 0 & 0.0 & $-5.2-71.0$ & 0.0 \\
\hline Bosutinib & 1 & 16 & 0 & 0 & 0.0 & $-3.9-82.3$ & 0.0 \\
\hline Vorinostat & 1 & 1 & 1 & 0 & 0.0 & $-3.9-82.3$ & 0.0 \\
\hline Total & 692 & 5,068 & 80 & 20 & 2.9 & $1.9-4.5$ & 0.4 \\
\hline Oral anticancer drugs & 1,832 & 10,909 & 205 & 52 & 2.8 & $2.2-3.7$ & 0.5 \\
\hline
\end{tabular}

SAE, serious adverse effects; S-1, Tegafur/Oteracil/Gimeracil; CI, confidence interval. 


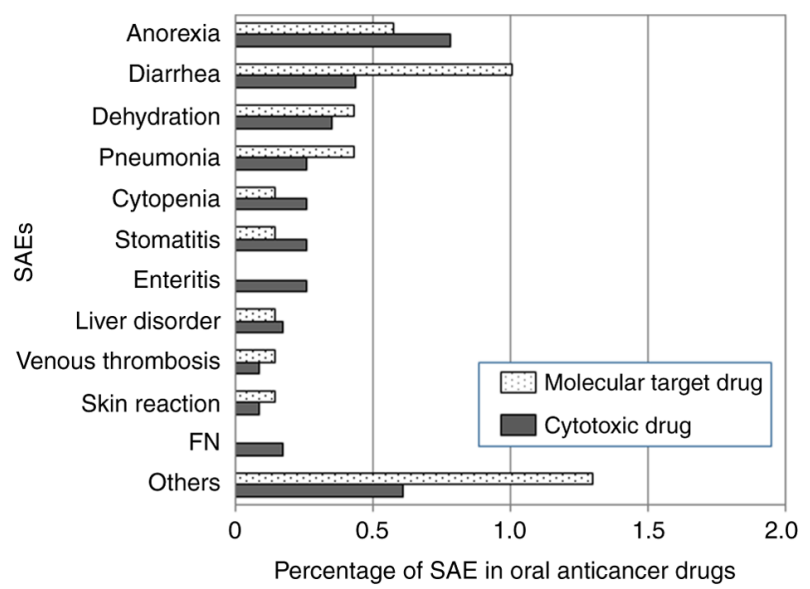

Figure 1. The effect of SAE with oral anticancer drugs. The percentage of SAE in patients treated with cytotoxic drugs $(n=1,140)$ and molecular target drugs $(n=692)$ are presented. SAE, serious adverse effect; FN, febrile neutropenia.

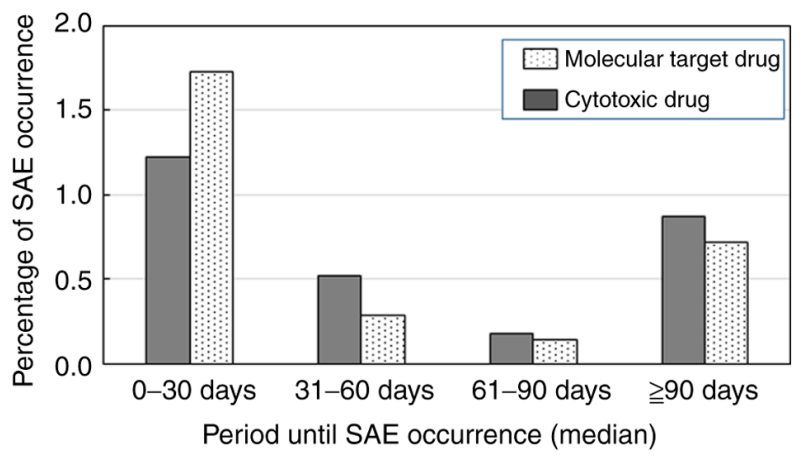

Figure 2. Median period until SAE occurrence after oral anticancer drug administration. The percentage of SAEs following cytotoxic anticancer $(n=1,140)$ and molecular target drug treatment $(n=692)$. SAE, serious adverse effect.

$61-90$ or $\geq 90$ days, the most frequent time to SAE was 0-30 days for both groups. In $65 \%$ of patients, SAEs occurred within 60 days of the start of the treatment.

Unplanned consultations for oral anticancer drugs. The total number of unplanned consultations for oral anticancer drugs was 100 (5.5\%; Table III). The number of unplanned consultations was $58(5.1 \%)$ for those on cytotoxic anticancer drugs and $42(6.1 \%)$ for those on molecular targeted anticancer drugs. Unplanned consultations were more frequent as a percentage of the total for those receiving molecular targeted anticancer drugs. Among patients receiving oral cytotoxic anticancer drugs with a high unplanned consultation occurrence, $40(6.0 \%)$ patients on S-1, 8 (3.9\%) patients on capecitabine and $5(6.5 \%)$ patients on Trifluridine/tipiracil had an unplanned consultation. For patients receiving molecular targeted anticancer drugs, 7 (10.8\%) patients on regorafenib, $7(5.3 \%)$ patients on gefitinib, $6(8.5 \%)$ patients on erlotinib and $5(5.1 \%)$ patients on imatinib had an unplanned consultation.

The nature of unplanned consultations for patients on cytotoxic anticancer drugs was $20(1.7 \%)$ reports of diarrhea, $12(1.0 \%)$ reports of nausea/vomiting, $12(1.0 \%)$ reports of skin

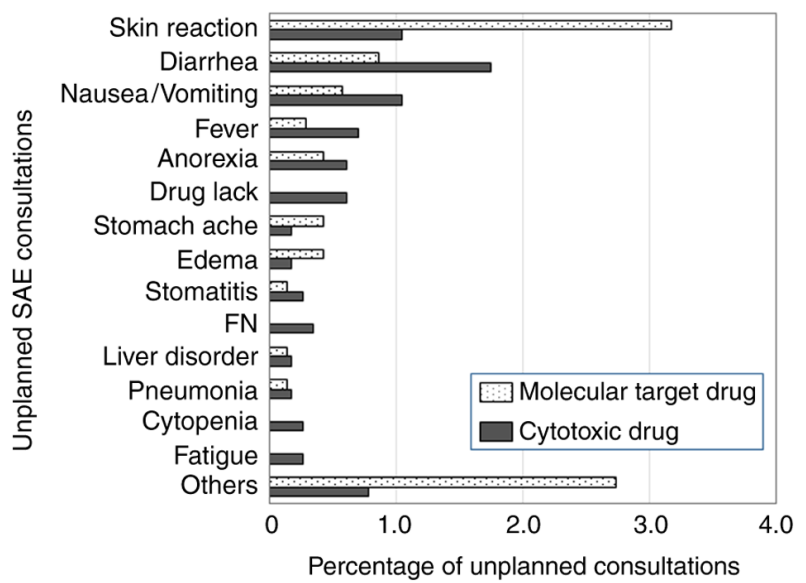

Figure 3. Unplanned consultations following oral anticancer drug administration. The percentage of unplanned consultations following the administration of cytotoxic $(n=1,140)$ and molecular target drugs $(n=692)$. FN, febrile neutropenia; Drug lack, unplanned consultation due to the lack of prescribed supportive care medication.

reactions, $8(0.7 \%)$ reports of fever and $7(0.7 \%)$ reports of anorexia. The nature of unplanned consultations for those on molecular targeted anticancer drugs was $22(3.2 \%)$ reports of skin reaction, $6(0.9 \%)$ reports of diarrhea and $4(0.6 \%)$ reports of anorexia (Fig. 3). The nature of unplanned consultations are shown in Table SII, and patients experienced $\leq 2$ types consistently. Unplanned consultations were primarily due to gastrointestinal toxicity for cytotoxic anticancer drugs and skin reactions for molecular targeted anticancer drugs. Unplanned consultations were $11(21.2 \%)$ in the SAEs group and $89(5.0 \%)$ in the non SAEs group, which was significantly higher in the SAE group $(\mathrm{P}<0.001)$.

Telephone calls from patients receiving oral anticancer drugs. The number of telephone calls from patients seeking medical advice during oral anticancer drug treatment was 1,412 calls from 694 patients, or $37.9 \%$ of all patients. For cytotoxic anticancer drugs, 852 telephone calls were made from 427 (37.5\%) patients and 560 calls from the 267 (38.5\%) patients on molecular targeted cancer drugs. The five most common reasons for calling for patients on cytotoxic anticancer drugs were fever, anorexia, diarrhea, nausea/vomiting and pain, whereas in patients on molecular targeted anticancer drugs they were fever, anorexia, diarrhea, skin disorder and pain. Of the 1,412 telephone calls, $152(10.8 \%)$ were unplanned consultations and $52(3.7 \%)$ were SAEs that required emergency hospitalization. Of the 152 unplanned consultations, 52 SAEs are included, as the physician determined the necessity of emergency hospitalization following the unplanned consultation.

\section{Discussion}

The present study focused on oral monotherapy anticancer drug treatment or combination therapy with oral and injectable anticancer drugs. Previous reports of SAEs in outpatient chemotherapy in Japan only include a survey of $\sim 50,000$ patients at 10 major cancer treatment hospitals in 2009 (7), and a small-scale survey of 158 patients in 2007 (16). 
Table III. Incidence rate of unplanned patient consultations following oral anticancer drug treatment according to the mechanism of action per patient and prescriptions.

A, Cytotoxic anti-cancer drugs

\begin{tabular}{|c|c|c|c|c|c|c|}
\hline Drug & Patients & Prescriptions & $\begin{array}{l}\text { Unplanned } \\
\text { consultations }\end{array}$ & $\begin{array}{l}\text { Unplanned } \\
\text { consultations/ } \\
\text { patients }(\%)\end{array}$ & $95 \%$ CI & $\begin{array}{c}\text { Unplanned } \\
\text { consultations/ } \\
\text { prescriptions (\%) }\end{array}$ \\
\hline S-1 & 670 & 3,438 & 40 & 6.0 & $4.4-8.0$ & 1.2 \\
\hline Capecitabine & 203 & 1,180 & 8 & 3.9 & $1.9-7.7$ & 0.7 \\
\hline Tegafur/Uracil & 122 & 457 & 1 & 0.8 & $-0.3-5.0$ & 0.2 \\
\hline $\begin{array}{l}\text { Trifluridine/Tipyrazyl } \\
\text { hydrochloride }\end{array}$ & 77 & 346 & 5 & 6.5 & $2.5-14.5$ & 1.4 \\
\hline Etoposide & 14 & 70 & 0 & 0.0 & $-3.6-25.2$ & 0.0 \\
\hline Melphalan & 17 & 84 & 2 & 11.8 & $2.0-35.6$ & 2.4 \\
\hline Hydroxycarbamide & 14 & 172 & 1 & 7.1 & $-0.8-33.5$ & 0.6 \\
\hline Cyclophosphamide & 10 & 44 & 0 & 0.0 & $-4.3-32.1$ & 0.0 \\
\hline Fludarabine & 4 & 5 & 0 & 0.0 & $-5.6-54.6$ & 0.0 \\
\hline Mercaptopurine & 4 & 31 & 0 & 0.0 & $-5.6-54.6$ & 0.0 \\
\hline Procarbazine & 4 & 9 & 1 & 25.0 & $3.4-71.1$ & 11.1 \\
\hline Temozomide & 1 & 5 & 0 & 0.0 & $-4.0-83.2$ & 0.0 \\
\hline Total & 1,140 & 5,841 & 58 & 5.1 & $4.0-6.5$ & 1.0 \\
\hline
\end{tabular}

B, Molecular targeted drugs

\begin{tabular}{|c|c|c|c|c|c|c|}
\hline Drug & Patients & Prescriptions & $\begin{array}{l}\text { Unplanned } \\
\text { consultations }\end{array}$ & $\begin{array}{l}\text { Unplanned } \\
\text { consultations/ } \\
\text { patients }(\%)\end{array}$ & $95 \% \mathrm{CI}$ & $\begin{array}{c}\text { Unplanned } \\
\text { consultations/ } \\
\text { prescriptions (\%) }\end{array}$ \\
\hline Gefitinib & 133 & 1,030 & 7 & 5.3 & $2.4-10.7$ & 0.7 \\
\hline Imatinib & 99 & 620 & 5 & 5.1 & $1.9-11.6$ & 0.8 \\
\hline Erlotinib & 71 & 464 & 6 & 8.5 & $3.6-17.6$ & 1.3 \\
\hline Regorafenib & 65 & 330 & 7 & 10.8 & $5.0-20.9$ & 2.1 \\
\hline Sorafenib & 48 & 353 & 0 & 0.0 & $-1.4-8.9$ & 0.0 \\
\hline Afatinib & 42 & 320 & 3 & 7.1 & $1.8-19.7$ & 0.9 \\
\hline Everolimus & 39 & 240 & 1 & 2.6 & $-0.7-14.4$ & 0.4 \\
\hline Lenvatinib & 25 & 225 & 3 & 12.0 & $3.3-30.8$ & 1.3 \\
\hline Sunitinib & 23 & 156 & 1 & 4.3 & $-0.9-22.7$ & 0.6 \\
\hline Lenalidomide & 23 & 152 & 2 & 8.7 & $1.3-28.0$ & 1.3 \\
\hline Pazopanib & 19 & 108 & 1 & 5.3 & $-0.9-26.5$ & 0.9 \\
\hline Axitinib & 19 & 129 & 3 & 15.8 & 4.7-38.4 & 2.3 \\
\hline Alectinib & 19 & 536 & 0 & 0.0 & $-3.0-19.8$ & 0.0 \\
\hline Crizotinib & 19 & 121 & 0 & 0.0 & $-3.0-19.8$ & 0.0 \\
\hline Nilotinib & 15 & 85 & 1 & 6.7 & $-0.8-31.8$ & 1.2 \\
\hline Thalidomide & 9 & 87 & 1 & 11.1 & $-0.2-45.7$ & 1.1 \\
\hline Lapatinib & 8 & 33 & 0 & 0.0 & $-4.8-37.2$ & 0.0 \\
\hline Dasatinib & 7 & 46 & 0 & 0.0 & $-5.0-40.4$ & 0.0 \\
\hline Tretinoin & 4 & 13 & 0 & 0.0 & $-5.6-54.6$ & 0.0 \\
\hline Tamibarotene & 2 & 3 & 1 & 50.0 & $9.5-90.6$ & 33.3 \\
\hline Bosutinib & 1 & 16 & 0 & 0.0 & $-3.9-83.3$ & 0.0 \\
\hline Vorinostat & 1 & 1 & 0 & 0.0 & $-3.9-83.3$ & 0.0 \\
\hline Total & 692 & 5,068 & 42 & 6.1 & $4.5-8.1$ & 0.8 \\
\hline Oral anticancer drugs & 1,832 & 10,909 & 100 & 5.5 & $4.5-6.6$ & 0.9 \\
\hline
\end{tabular}

S-1, Tegafur/Oteracil/Gimeracil; CI, confidence interval. 
Several studies on emergency hospitalizations due to SAEs caused by outpatient chemotherapy have been performed in other countries (5-6,17-20), and this may be due the increased popularity of outpatient chemotherapy in other countries compared with Japan. Injectable drugs are the primary option for treating cancer in Japan, and there are no previous reports from Japan regarding oral anticancer drug safety, to the best of our knowledge.

The age and performance status of the patient population in the present study was consistent with the recommendations for oral anticancer drug treatments used in Japan, making the present study less biased towards specific types of cancer. In the present study, the rate of SAEs was $2.8 \%$ for all patients and $0.5 \%$ for all oral anticancer drug prescriptions, which was similar to the overall prescription rate of $0.5-2.8 \%$ in a multicenter survey primarily using injectable drug treatment (Table II) (7). As opposed to oral anticancer drug monotherapy, combination therapy consisting of injectable and oral anticancer drugs generally use multiple anticancer drugs, increasing the likelihood of serious side effects. Despite the use of primarily oral anticancer drug monotherapy, the reason for the SAE rate being similar in the present to that of injectable anticancer drugs may be due to the fact that $\mathrm{S}-1$ has been reported to have a high SAE frequency in Japan (7), and new molecular targeted anticancer drugs with serious side effects were also included in the present study. In similar studies performed in other countries (5-6,17-21), reported SAE rates were 7.4-45\%, all higher than the SAE rate reported in the present study. This discrepancy may be due to differences in the definitions of SAEs between countries, in reports of pre- and post-operative chemotherapy for breast cancer patients $(6,20)$, and those identifying causality based on information extracted from public databases for patients hospitalized with emergencies $(6,17)$.

Although it was reported in a multicenter survey in Japan that the SAE frequency for S-1 (3.3-5.0\%) tends to be higher than that of other drugs (7), the SAE rate for S-1 was found to be similar to that of the other oral anticancer drugs used in the present study. A clinical trial (ACTS-GC) (22) showed the efficacy of S-1 as an adjuvant chemotherapy for gastric cancer, and while the SAE frequency was unknown, the non-hematological toxicity of grade 3 or higher was $0.2-6.0 \%$. Furthermore, in similar clinical trials of S-1 for advanced recurrent pancreatic cancer (23), non-hematological toxicity of grade 3 or higher was reported as 5.1-13.6\%. Clinical trials are performed according to eligibility criteria; thus, it is perhaps not surprising that more adverse events occur in patients who do not meet the eligibility criteria (8). It is hypothesized that there may be two factors that contributed to the SAE frequency in the present study being the same or lower than that of clinical trials. First, the management of side effects by doctors at our hospital has been improved by them participating in drug development clinical trials. Second, since 2009, when a previous study was performed, a patient support system for patients treated with oral anticancer drugs has been introduced, with patients being interviewed by an outpatient pharmacist and a nurse before consulting a physician. An emergency telephone consultation service during treatment is also provided. Thus, it is hypothesized that our outpatient chemotherapy support system has been strengthened by the active involvement of medical staff.

The nature of SAEs reported are primarily gastrointestinal toxicity $(17,21)$, fever and infection $(6,18,20,21)$. The nature of SAEs that occurred in the present study were similar to those of previous reports of anorexia due to cytotoxic anticancer drugs, or diarrhea, and other gastrointestinal symptoms due to molecular targeted anticancer drugs $(17,21)$. Therefore, further studies on the treatments of gastrointestinal complications are warranted. One potential explanation for the few reports of fever and infection is that the present study included oral fluorinated pyrimidine drugs (cytotoxic anticancer drugs) and molecular targeted anticancer drugs associated with reduced bone marrow suppression. When the SAEs that occurred were classified by CTCAE, serious symptoms of grade 3 or higher accounted for $48.2 \%$, accounting for half of all cases. However, $32.7 \%$ of cases had an unknown grade evaluation, which is expected to be more frequent.

The median time to SAE occurrence was 32 days, which was similar to previously reported results of $>30$ days $(17,21)$. One reason for this could be that gastrointestinal symptoms, such as anorexia and diarrhea, are likely to occur early on during anticancer drug treatment (24). Early gastrointestinal SAEs may be mitigated by reducing the dose of drugs and providing supportive care while continuing treatment. The median time to SAE occurrence for molecular targeted anticancer agents (16 days) was shorter than that for cytotoxic drugs (45 days). Among molecular targeted anticancer drugs, there have been reports of early occurrence of severe diarrhea with afatinib (25) and lapatinib (26), as well as gastrointestinal bleeding and erythema multiforme with regorafenib (27). Therefore, it is possible that SAEs occur earlier for molecular targeted anticancer drugs than for cytotoxic drugs. Furthermore, as intervention by medical staff, such as pharmacists and nurses, has been reported to decrease the severity of side effects (9-12), the early management of side effects by various medical staff may be effective.

The frequency of unplanned consultations was twice that of SAE, and the causal drugs were similar to those causing SAEs (Table III). The reasons for unplanned consultations were similar to those for SAEs in cytotoxic anticancer drugs but, in molecular targeted anticancer drugs, skin reactions occurred most frequently. Skin reactions caused by molecular targeted anticancer drugs have been reported to reduce the patients' quality of life (28), and skin reactions can be expected to cause patients to seek medical attention. However, due to the lack of emergency and severity, few skin reactions develop into SAEs, and management with outpatient consultation is likely. It has been reported that patients who have an unplanned consultation may be at a higher risk of subsequent emergency hospitalization (19). The present study had similar results, so information obtained at unplanned consultations could possibly predict SAE occurrence.

In patients receiving oral anticancer drug treatments, $\sim 40 \%$ sought medical information through telephone calls. The primary reason for these calls was fever during treatment, which was different from the primary reasons for SAE or unplanned consultations. Although the reasons for fever vary and can be difficult to identify, the most concerning type of fever during cancer chemotherapy is febrile neutropenia (FN). 
If the Multinational Association for Supportive Care in Cancer (MASCC) score indicates low risk, treatment with oral antimicrobial agents is optional in FN (29). Several patients on oral anticancer drug therapy are classified as low risk. In our hospital, oral antimicrobials are prescribed at the start of anticancer drug therapy in several cases to protect against FN. If fever develops at home, patients can promptly start treatment with antimicrobial drugs after a telephone call to the hospital without the need for an unplanned consultation or emergency hospitalization. As several subjects undergoing outpatient chemotherapy have a low risk of developing FN, it is conceivable that they could successfully recover from the fever at home.

A limitation of the present study was that it was conducted based on a single institution (the National Cancer Center Hospital East) and therefore it does not reflect the SAEs for oral anticancer drug treatment occurring throughout Japan. In addition, this was a retrospective study with missing data, such as patient background and causality. However, missing data and loss of patients to follow up accounted for $<1 \%$ of all patients, so the results of this study will not be significantly affected by the inclusion of these data. The incidence rate of SAE was accurate, as SAE cases were deliberated and recorded at conferences after emergency hospitalization. However, unplanned consultations and telephone calls were only judged by the attending physician, and it was possible that there were causal relationships and missing data, so they may have been underestimated. Certain oral anticancer drugs were combined with injectable anticancer drugs, and it was not possible to distinguish which drug resulted in the SAE. The Kaplan-Meier curves are an effective analytical method for showing the SAE occurrence period. We tried to show SAE occurrence period using Kaplan-Meier curves, classified by mechanism of action, but it was difficult to detect statistical differences in the initial period between each cohort. Therefore, it needs to be clarified in future studies. Due to the low frequency of SAEs, stratification analysis was not performed in this study. Finally, the adopted definition of SAE herein was the same as that used in a multicenter survey from 2009 (7), making direct comparisons with other previously published reports that may have used different definitions of SAE difficult.

In conclusion, the present study is the first to analyze SAEs due to oral anticancer drug therapy in clinical practice in Japan. The frequency of SAE occurrence was similar to that of previous surveys for injection-based cancer drugs. SAEs, particularly gastrointestinal toxicity, often occurred within 30 days of the start of treatment. The importance of monitoring patients for gastrointestinal toxicity and advocating for patient education by a team of medical staff should be highlighted. In future studies, risk factors for SAEs caused by oral anticancer drug therapy should be identified and trials should assess interventions in patients with a high risk of exhibiting a SAE.

\section{Acknowledgements}

Not applicable.

\section{Funding}

No funding was received.

\section{Availability of data and materials}

The datasets used and/or analyzed during the current study are available from the corresponding author on reasonable request.

\section{Authors' contributions}

KK conceived the current study. RM, YK, MY and TA made substantial contributions to the conception and design of the present study. KK, AK and YK collected and analyzed the data from medical records. KK wrote the manuscript. RM, YK, MY and TA provided editorial review and contributed to discussions. All the authors read and approved the final version of the manuscript.

\section{Ethics approval and consent to participate}

The present study was approved by the National Cancer Center Hospital East Institutional Review Board (approval no. 2016-077) and Tokyo University of Science Institutional Review Board (approval no. 16032).

\section{Patient consent for publication}

Not applicable.

\section{Competing interests}

The authors declare that they have no competing interests.

\section{References}

1. Weingart SN, Brown E, Bach PB, Eng K, Johnson SA, Kuzel TM, Langbaum TS, Leedy RD, Muller RJ, Newcomer LN, et al: NCCN Task Force Report: Oral chemotherapy. J Natl Compr Canc Netw 6 (Suppl 3): S1-S14, 2008.

2. Timmers L, Beckeringh JJ, van Herk-Sukel MP, Boven E and Hugtenburg JG: Use and costs of oral anticancer agents in the Netherlands in the period 2000-2008. Pharmacoepidemiol Drug Saf 21: 1036-1044, 2012.

3. Mancini R, Kaster M, Vu B, Modlin J and Wilson B: Implementation of a pharmacist managed interdisciplinary oral chemotherapy program in a community cancer center. J Hematol Oncol Pharm 1: 23-30, 2011.

4. Twelves C, Gollins S, Grieve R and Samuel L: A randomised cross-over trial comparing patient preference for oral capecitabine and 5-fluorouracil/leucovorin regimens in patients with advanced colorectal cancer. Ann Oncol 17: 239-245, 2006.

5. Brooks GA, Kansagra AJ, Rao SR, Weitzman JI, Linden EA and Jacobson JO: A clinical prediction model to assess risk for chemotherapy-related hospitalization in patients initiating palliative chemotherapy. JAMA Oncol 1: 441-447, 2015.

6. Hassett MJ, O'Malley AJ, Pakes JR, Newhouse JP and Earle CC: Frequency and cost of chemotherapy-related serious adverse effects in a population sample of women with breast cancer. J Natl Cancer Inst 98: 1108-1117, 2006.

7. Koich G: Outpatient management of anticancer drug trials and the current state of outpatient chemotherapy in Japan. Pharma Medica 28: 61-65, 2010 (In Japanese).

8. Prince RM, Atenafu EG and Krzyzanowska MK: Hospitalizations during systemic therapy for metastatic lung cancer: A systematic review of real world vs clinical trial outcomes. JAMA Oncol 1: 1333-1339, 2015.

9. Stein J and Mann J: Specialty pharmacy services for patients receiving oral medications for solid tumors. Am J Health Syst Pharm 73: 775-796, 2016. 
10. Patel JM, Holle LM, Clement JM, Bunz T, Niemann C, Chamberlin KW and Chamberlin KW: Impact of a pharmacist-led oral chemotherapy-monitoring program in patients with metastatic castrate-resistant prostate cancer. J Oncol Pharm Pract 22: 777-783, 2016.

11. Lam MS and Cheung N: Impact of oncology pharmacist-managed oral anticancer therapy in patients with chronic myelogenous leukemia. J Oncol Pharm Pract 22: 741-748, 2016.

12. Schneider SM, Adams DB and Gosselin T: A tailored nurse coaching intervention for oral chemotherapy adherence. J Adv Pract Oncol 5: 163-172, 2014.

13. Kimura M, Go M, Iwai M, Usami E and Yoshimura T: Evaluation of the role and usefulness of a pharmacist outpatient service for patients undergoing monotherapy with oral anti-cancer agents J Oncol Pharm Pract 23: 413-421, 2017.

14. Arakawa-Todo M, Yoshizawa T, Zennami K, Nishikawa G, Kato Y, Kobayashi I, Kajikawa K, Yamada Y, Matsuura K, Tsukiyama I, et al: Management of adverse events in patients with metastatic renal cell carcinoma treated with sunitinib and clinical outcomes. Anticancer Res 33: 5043-5050, 2013.

15. NCI Guidelines for Investigators: Adverse event reporting requirements for DCTD (CTEP and CIP) and DCP INDs and IDEs. Page 5. https://ctep.cancer.gov/protocolDevelopment/ electronic_applications/docs/aeguidelines.pdf. Accessed February 29,2012

16. Uramoto H, Iwashige A, Kagami S and Tsukada J: Prediction of emergency hospitalization of outpatients receiving cancer chemotherapy. Anticancer Res 27: 1133-1136, 2007.

17. McKenzie H, Hayes L, White K, Cox K, Fethney J, Boughton M and Dunn J: Chemotherapy outpatients' unplanned presentations to hospital: A retrospective study. Support Care Cancer 19: 963-969, 2011

18. Rashid N, Koh HA, Baca HC, Li Z, Malecha S, Abidoye O and Masaquel A: Clinical impact of chemotherapy-related adverse events in patients with metastatic breast cancer in an integrated health care system. J Manag Care Spec Pharm 21: 863-871, 2015.

19. Aprile G, Pisa FE, Follador A, Foltran L, De Pauli F, Mazzer M Lutrino S, Sacco CS, Mansutti M and Fasola G: Unplanned presentations of cancer outpatients: A retrospective cohort study. Support Care Cancer 21: 397-404, 2013.

20. Pittman NM, Hopman WM and Mates M: Emergency room visits and hospital admission rates after curative chemotherapy for breast cancer. J Oncol Pract 11: 120-125, 2015.
21. Hassett MJ, Rao SR, Brozovic S, Stahl JE, Schwartz JH, Maloney B and Jacobson JO: Chemotherapy-related hospitalization among community cancer center patients. Oncologist 16: 378-387, 2011.

22. Sakuramoto $S$, Sasako M, Yamaguchi $T$, Kinoshita $T$, Fujii M, Nashimoto A, Furukawa H, Nakajima T, Ohashi Y, Imamura $\mathrm{H}$, et al: Adjuvant chemotherapy for gastric cancer with S-1, an oral fluoropyrimidine. N Engl J Med 357: 1810-1820, 2007.

23. Okusaka T, Funakoshi A, Furuse J, Boku N, Yamao K, Ohkawa S and Saito H: A late phase II study of S-1 for metastatic pancreatic cancer. Cancer Chemother Pharmacol 61: 615-621, 2008.

24. Naeim A, Dy SM, Lorenz KA, Sanati H, Walling A and Asch SM: Evidence-based recommendations for cancer nausea and vomiting. J Clin Oncol 26: 3903-3910, 2008.

25. Sequist LV, Yang JC, Yamamoto N, O'Byrne K, Hirsh V, Mok T, Geater SL, Orlov S, Tsai CM, Boyer M, et al: Phase III study of afatinib or cisplatin plus pemetrexed in patients with metastatic lung adenocarcinoma with EGFR mutations. J Clin Oncol 31: 3327-3334, 2013

26. Geyer CE, Forster J, Lindquist D, Chan S, Romieu CG, Pienkowski T, Jagiello-Gruszfeld A, Crown J, Chan A, Kaufman B, et al: Lapatinib plus capecitabine for HER2-positive advanced breast cancer. N Engl J Med 355: 2733-2743, 2006.

27. Grothey A, Van Cutsem E, Sobrero A, Siena S, Falcone A, Ychou M, Humblet Y, Bouché O, Mineur L, Barone C, et al: Regorafenib monotherapy for previously treated metastatic colorectal cancer (CORRECT): An international, multicentre, randomised, placebo-controlled, phase 3 trial. Lancet 381: 303-312, 2013.

28. Kaori N, Fumiko S, Eiko K and Naoko S: Relationship between Symptom Experience and QOL for Cancer Outpatients Receiving Molecular Targeted Therapy. J Jpn Soc Cancer Nursing 28: 5-12, 2014.

29. Taplitz RA, Kennedy EB and Flowers CR: Outpatient management of fever and neutropenia in adults treated for malignancy: American society of clinical oncology and infectious diseases society of america clinical practice guideline update summary. J Oncol Pract 14: 250-255, 2018. 